\title{
Evaluation of Genetic Analysis with Autosomal Recessive Bestrophinopathy
}

\author{
Ali Mert Kocer, Mehmet Yasin Teke, Furkan Emre Sogut \\ Department of Ophthalmology, Ulucanlar Eye Education and Research Hospital, Ankara, Turkey
}

Purpose: We aimed to report a patient with autosomal recessive bestrophinopathy (ARB) who have novel compound heterozygous mutations in BEST1.

Case summary: A 35-year old male patient who had no history of known trauma and disease was admitted to our hospital with bilateral visual disturbance since his childhood. Dilated fundus examination revealed widespread yellow-white subretinal accumulation and white scar formation on both posterior poles. Hyperfluorescent foci were observed along the vessel arcades in fundus autofluorescence imaging. Optical coherence tomography showed serous subretinal fluid under the bilateral macula. Arden rate was found to be 1.32 for right eye and 1.28 for left eye in the electrooculography examination. Bestrophinopathy was considered in the patient and a genetic examination test was performed for BEST1 gene. The whole gene sequence analysis test (Miseq-Illumina) performed on the BEST1 gene of the patient resulted in c.604C>T (p.Arg202Trp) (pR202W) /c.1013G>A (p.Trp338 ${ }^{*}$ ) (p.W338*) compound heterozygous mutation.

Conclusions: ARB is a rare disease and usually associated with consanguineous marriage, the disease may also occur with different mutations of both spouses. For this reason, it is important that ophthalmologists consider the possibility of best retinopathy even in the absence of a consanguineous history.

Keywords: Autosomal recessive; Bestrophinopathy; BEST1 gene; Bestrophin-1; Heterozygous; Mutation

\section{Introduction}

The BEST1 gene, which encodes the transmembrane protein named bestrophin-1 is located on the long arm of the chromosome 11 and consists of 585 amino acids [1]. Bestrophin-1 is found in the basolateral plasma membrane of retinal pigment epithelium (RPE) in healthy individuals and is a multifunctional protein in RPE [2]. Bestrophin-1 protein plays a role in transepithelial chloride flow by regulating the func- tion of voltage-dependent $\mathrm{Ca}^{2+}$ channels within the cell [3-5]. Abnormal chlorine formation due to the deterioration of this function disrupts RPE fluid transfer and causes lipofuscin deposition. Demonstration of the mutation in the BEST1 gene is essential for the definitive diagnosis of Bestrophinopathy. We aimed to report a patient with autosomal recessive bestrophinopathy (ARB) who have novel compound heterozygous mutations in BEST1.

\section{Address reprint requests to Ali Mert Kocer, MD}

Ulucanlar Eye Education and Research Hospital, \#59 Ulucanlar Cd., Altındağ, Ankara 06250, Turkey

Tel: 90-0506-222-8555, Fax: 90-0312-312-3805

E-mail: alimertkocer@gmail.com
Received: 2019. 3. 14

Revised: 2019. 5. 4

Accepted: 2019.5 .20 


\section{Case Report}

A 35-year-old male patient who had no history of known trauma and disease was admitted to our hospital with bilateral visual disturbance since his childhood. The patient was followed up with the diagnosis of Stargardt's disease and chronic central serous chorioretinopathy. The patient did not have a family history of low vision and close consanguineous marriage. Bilateral 3.0 diopters hypermetropic refraction was detected on the patient's examination. The patient's best corrected visual acuity was bilateral 0.1 at the Snellen chart. There was no pathology in the anterior segment examination. Dilated fundus examination revealed widespread yellow-white subretinal accumulation and white scar formation on both posterior poles (Fig. 1). Hyperfluorescent foci were observed along the vascular arcades in fundus autofluorescence (HRA2, Heidelberg Engineering, Heidelberg, Germany) imaging (Fig. 2). Spectral domain optical coherence tomography (SD OCT; Spectralis, Heidelberg Engineering) showed serous subretinal fluid under the bilateral macula (Fig. 3). Arden rate was found to be 1.32 for right eye and 1.28 for left eye in the electrooculography (EOG) examination (Fig. 4). Bestrophinopathy was considered in the patient and a genetic examination test was performed for BEST1 gene. We adhered to the principles of the Declaration of Helsinki

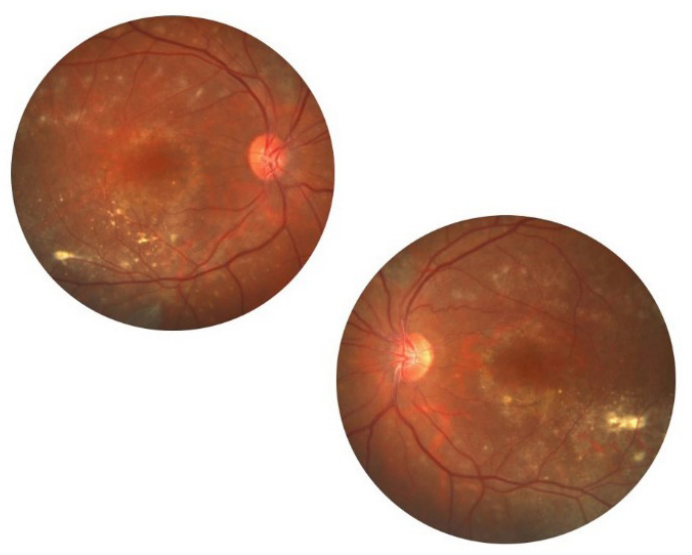

Figure 1. Dilated fundus examination reveals widespread yellow-white subretinal accumulation and white scar formation on both posterior poles. Subretinal yellow-white deposits are observed especially at the vascular arcades and scar formation locate at the extrafoveal area on both retina. Subretinal deposits (lipofuscin) appears to be an accumulation of debris between retinal pigment epithelium and neuroretina. in this case report. Additionally patient read and signed the informed consent before the genetic investigation. The whole gene sequence analysis test (Miseq-Illumina) performed on the BEST1 gene of the patient resulted in c.604C $>\mathrm{T}$ (p.Arg202Trp) $(\mathrm{pR} 202 \mathrm{~W}) / \mathrm{c} .1013 \mathrm{G}>\mathrm{A}\left(\mathrm{p} \cdot \operatorname{Trp} 338^{*}\right)\left(\mathrm{p} \cdot \mathrm{W} 338^{*}\right)$ compound heterozygous mutation. In the genetic analysis report, it was stated that the mutation c.604C $>\mathrm{T}$ (p.Arg202Trp) (pR202W) was previously related to the disease, but the mutation c.1013G > A (p.Trp338*) (p.W338*) was not previously

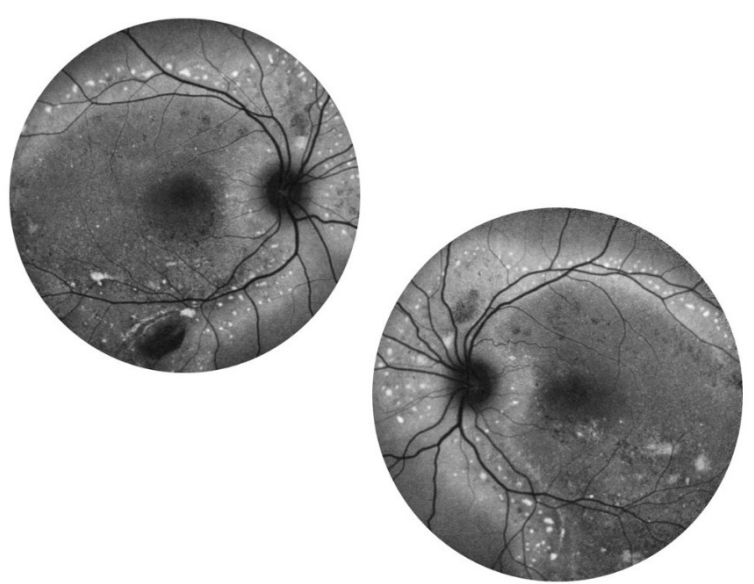

Figure 2. Hyperfluorescent foci are observed along the vascular arcades and around the optic disc in fundus autofluorescence (FAF). These hyperfluorescent foci are observed at the same area with the subretinal deposits. FAF image showing that macula is hypoautofluorescent because of serous subretinal fluid. Additionally, scar formations show hypoautofluorescent appearance.

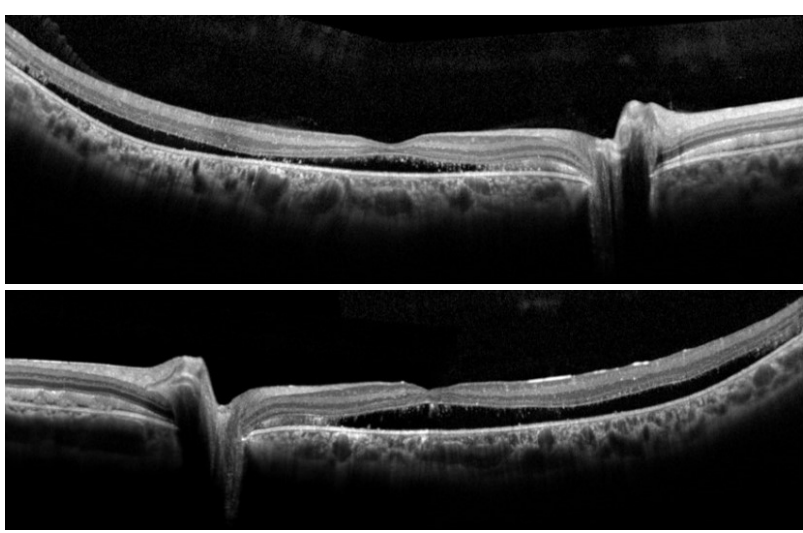

Figure 3. Optical coherence tomography shows serous neuroretinal detachment under the bilateral macula with some accumulation of photoreceptor outer segment debris. 
$(\mu \mathrm{V})$

\section{RE}

Minimum $=106 \mu \mathrm{V}$

Maximum $=139 \mu \mathrm{V}$

ARDEN ratio $=132 \%$

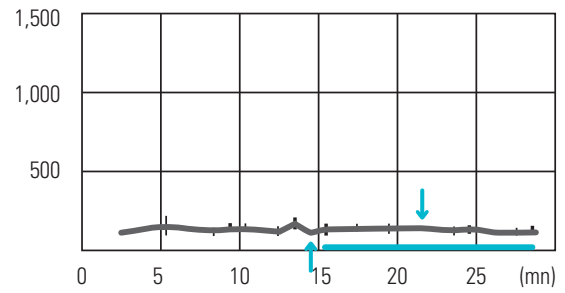

$(\mu \mathrm{V})$

LE

Minimum $=107 \mu \mathrm{V}$

Maximum $=134 \mu \mathrm{V}$

ARDEN ratio $=126 \%$

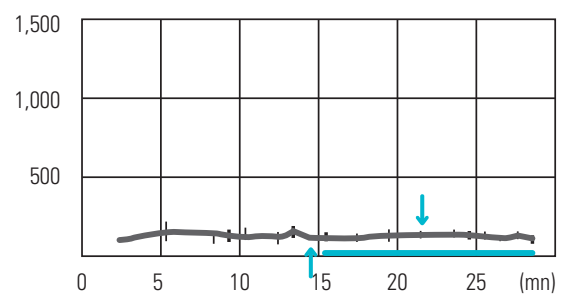

Figure 4. Electro-oculogram shows subnormal Arden ratio which is between 1.2-1.5. Upward arrow indicates the minimum amplitude during the dark phase and downward arrow indicates the maximum amplitude during the light phase. $\mathrm{RE}=$ right eye; $\mathrm{LE}=$ left eye.

reported in relation to the disease. This mutation was also reported as a cause of the disease due to early stop codon formation according to mutation taster program analysis from the in silico evaluation tools.

\section{Discussion}

Bestrophinopathy group includes the best vitelliform macular dystrophy, adult-onset vitelliform macular dystrophy, autosomal dominant vitreoretinochoroidopathy, and retinitis pigmentosa and $\mathrm{ARB}$. ARB which is included in this group of diseases was first described in 2008 by Burgess et al. [3] and has been shown to be autosome recessive inheritance. ARB is characterized by the total absence of the bestrophin-1 protein (mutation in two alleles). Bilateral retinal involvement is observed in ARB and usually becomes symptomatic in the first two decades. The main affected area is the mid-peripheral and posterior retinal area and yellowish vitelliform lesions are seen. Serous subretinal fluid and cystic macular changes can also be observed. This fluid accumulation observed in macula does not respond to systemic steroids with topical carbonic anhydrase inhibitors. It is usually accompanied by additional ocular disorders such as hypermetropia, short axial length, amblyopia and angle-clo- sure glaucoma [3]. Visual acuity in the course of the disease is relatively stable. Vitelliform lesions may increase or decrease over time. Choroidal neovascular membrane may develop. EOG is an important test for the diagnosis, follow-up and detect carrier of best disease. EOG is used to detect very mild anomalies especially in patients with normal fundus examinations or to assess carriers in families with suspected disease and to evaluate individuals in whom disease has not yet occurred. But also, opposed to autosomal dominant best disease, the parents in many ARB cases do not have fundus findings and their EOG is normal [6]. Anomalous response can be observed in EOG in conditions such as Best disease and carriers, retinitis pigmentosa, toxic retinopathies such as chloroquine use, choroideremia and common choroiditis. In ARB, EOG is significantly impaired. The increase in potential against light is below normal in ARB patients. But, as a normal EOG may occur in the presence of a classical fundus appearance [7]. Electroretinogram shows relative normal responses in early stages of disease. There may be delay in scotopic and photopic responses in follow-up. In the differential diagnosis a large group of diseases such as hereditary drusen, adult onset Stargardt macula dystrophy, multifocal pattern dystrophy (pseudo-Stargardt), adult onset vitelliform macular dystrophy, MIDD retinal dystrophy (maternally inherited diabetes and deafness), chronic central serous chorioretinopathy, acute exudative polymorphosis vitelliform maculopathy, paraneoplastic exudative vitelliform retinopathy, chorioretinitis, lymphoma and best vitelliform macular dystrophy should be considered.

Demonstration of the mutation in the BEST1 gene is essential for the definitive diagnosis of Bestrophinopathy. More than 200 mutations are shown in the BEST1 gene [5,8]. In our case, genetic analysis (Miseq-Illumina) revealed c.604C $>\mathrm{T}$ (p.Arg202Trp) (pR202W)/c.1013G > A (p.Trp338*) (p.W338*) combined heterozygote mutation. The mutation c.604C $>\mathrm{T}$ (p.Arg202Trp) (pR202W) was previously reported to be related to the disease and the mutation c.1013G $>$ A (p.Trp338*) (p.W338*) was not previously associated with the disease. This mutation was also reported as a cause of the disease due to early stop codon formation according to mutation taster program analysis from the in silico evaluation tools. BEST1 gene mutation is more likely to be associated with homozygous mutation due to consanguineous marriages [9]. In our patient, a mutation that was not previously reported was detected and also a combined heterozygote mutation 
was observed. The appearance of the combined heterozygote mutation shows that although the spouses are not related, both have different mutations. However, it was not possible to prove that two variants located in trans due to insufficient parent genetic testing. The mutation spectrum of the BESTI gene may differed in different population [10]. This finding may be completely coincidental or may be due to the higher prevalence of this disease and carriage in our country than expected.

Consequently, ARB is a rare disease characterized by common yellow-white subretinal deposits resulting from mutations in the BEST1 gene encoding bestrophin 1. Although it is usually associated with consanguineous marriage, the disease may also occur with different mutations of both spouses. For this reason, it is important that ophthalmologists consider the possibility of best retinopathy even in the absence of a consanguineous history.

\section{Conflicts of Interest}

The authors declare no conflicts of interest relevant to this article.

\section{References}

1. Marquardt A, Stöhr H, Passmore LA, et al. Mutations in a nove gene, VMD2, encoding a protein of unknown properties cause juvenile-onset vitelliform macular dystrophy (Best's disease). Hum Mol Genet 1998;7:1517-25.
2. Marmorstein AD, Kinnick TR, Stanton JB, et al. Bestrophin-1 influences transepithelial electrical properties and $\mathrm{Ca} 2+$ signaling in human retinal pigment epithelium. Mol Vis 2015;21:347-59.

3. Burgess R, Millar ID, Leroy BP, et al. Biallelic mutation of BEST1 causes a distinct retinopathy in humans. Am J Hum Genet 2008;82:19-31.

4. Johnson AA, Lee YS, Chadburn AJ, et al. Disease-causing mutations associated with four bestrophinopathies exhibit disparate effects on the localization, but not the oligomerization, of Bestrophin-1. Exp Eye Res 2014;121:74-85.

5. Pasquay C, Wang LF, Lorenz B, Preising MN. Bestrophin 1--phenotypes and functional aspects in bestrophinopathies. Ophthalmic Genet 2015;36:193-212.

6. Boon CJ, Klevering BJ, Leroy BP, et al. The spectrum of ocular phenotypes caused by mutations in the BEST1 gene. Prog Retin Eye Res 2009;28:187-205.

7. Khan KN, Islam F, Holder GE, et al. Normal electrooculography in best disease and autosomal recessive bestrophinopathy. Retina 2018;38:379-86.

8. Kinnick TR, Mullins RF, Dev S, et al. Autosomal recessive vitelliform macular dystrophy in a large cohort of vitelliform macular dystrophy patients. Retina 2011;31:581-95.

9. Piñeiro-Gallego T, Álvarez M, Pereiro I, et al. Clinical evaluation of two consanguineous families with homozygous mutations in BEST1. Mol Vis 2011;17:1607-17.

10. Tian L, Sun T, Xu K, et al. Screening of BEST1 gene in a Chinese cohort with best vitelliform macular dystrophy or autosomal recessive bestrophinopathy. Invest Ophthalmol Vis Sci 2017;58:3366-75. 